\title{
Seizures and Cardiomyopathy in a Patient with Pallister-Killian Syndrome due to Hexasomy 12p Mosaicism
}

\author{
Reha M. Toydemir ${ }^{a-c}$ Emanuele Panza $^{d}$ Maria C. Longhurst ${ }^{c}$ Sarah T. South $^{e}$ \\ Alan F. Rope \\ ${ }^{a}$ Department of Pathology, University of Utah, Salt Lake City, UT, USA; b Department of Pediatrics, University of Utah,

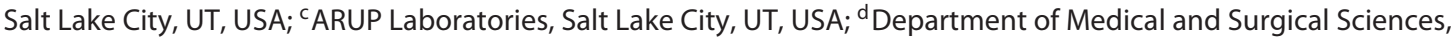 \\ University of Bologna, Bologna, Italy; ${ }^{\mathrm{e} A n c e s t r y}$, Salt Lake City, UT, USA; ${ }^{\mathrm{f}}$ Genome Medical, Portland, OR, USA
}

\author{
Keywords \\ Dysmorphic features · Hexasomy $12 p \cdot$ Mosaicism · Pallister- \\ Killian syndrome $\cdot$ Seizures
}

\begin{abstract}
Pallister-Killian syndrome (PKS) is a rare disorder presenting with developmental delay, numerous dysmorphic features, and skin pigmentation anomalies. It is caused by mosaic tetrasomy of the short arm of chromosome 12. In most instances, tetrasomy is due to a supernumerary isochromosome $\mathrm{i}(12)(\mathrm{p} 10)$. Although mitotic instability is a generally accepted behavior for supernumerary chromosomes, hexasomy $12 p$ due to a gain of an isochromosome $12 p$, has been hardly ever reported. We report a 10 year follow-up on a girl with 2 copies of isochromosome consisting of the short arm of chromosome 12, who has craniofacial features seen in PKS, such as sparse hair with an unusual pattern, sparse eyebrows, lacrimal duct stenosis, submucous cleft palate, Pallister lip (a relatively long philtrum continuing into the vermillion border of the upper lip), narrow palate, and wide alveolar ridges. She also has other abnormalities, including unilateral renal dysgenesis, rectovaginal fistula, pre-axial polydactyly of the right hand, severe global developmental delay, and hypotonia as well as some features suggestive of
\end{abstract}

\section{KARGER}

() 2020 S. Karger AG, Basel

karger@karger.com

www.karger.com/msy mosaicism such as bilateral asymmetry, patchy areas of rough skin, and retinal mottling. Initial cytogenetic studies from peripheral blood showed a normal female karyotype. Further cytogenetic studies on a skin biopsy showed mosaicism with 2 copies of the supernumerary isochromosome $12 \mathrm{p}$.

(c) 2020 S. Karger AG, Basel

Pallister-Killian syndrome (PKS; OMIM 601803) is a rare developmental disorder characterized by dysmorphic facial features, pigmentary anomalies of the skin, cardiac anomalies, diaphragmatic hernia, hypotonia, developmental delay, seizures, and intellectual disabilities [Schinzel, 1991; Kostanecka et al., 2012; Wilkens et al., 2012; Blyth et al., 2015; Costa et al., 2015]. In most instances, PKS is caused by mosaic tetrasomy of the short arm of chromosome 12, due to a supernumerary isochromosome i(12)(p10) [Schinzel , 1991; Kostanecka et al., 2012; Wilkens et al., 2012; Blyth et al., 2015; Costa et al., 2015].

Tissue-limited mosaicism for one isochromosome $12 \mathrm{p}$ is well-described in the literature [Van den Veyver et al., 1993; Choo et al., 2002; Vogel et al., 2009; Conlin et al., 2012; Aliu et al., 2013]. However, mosaicism for 

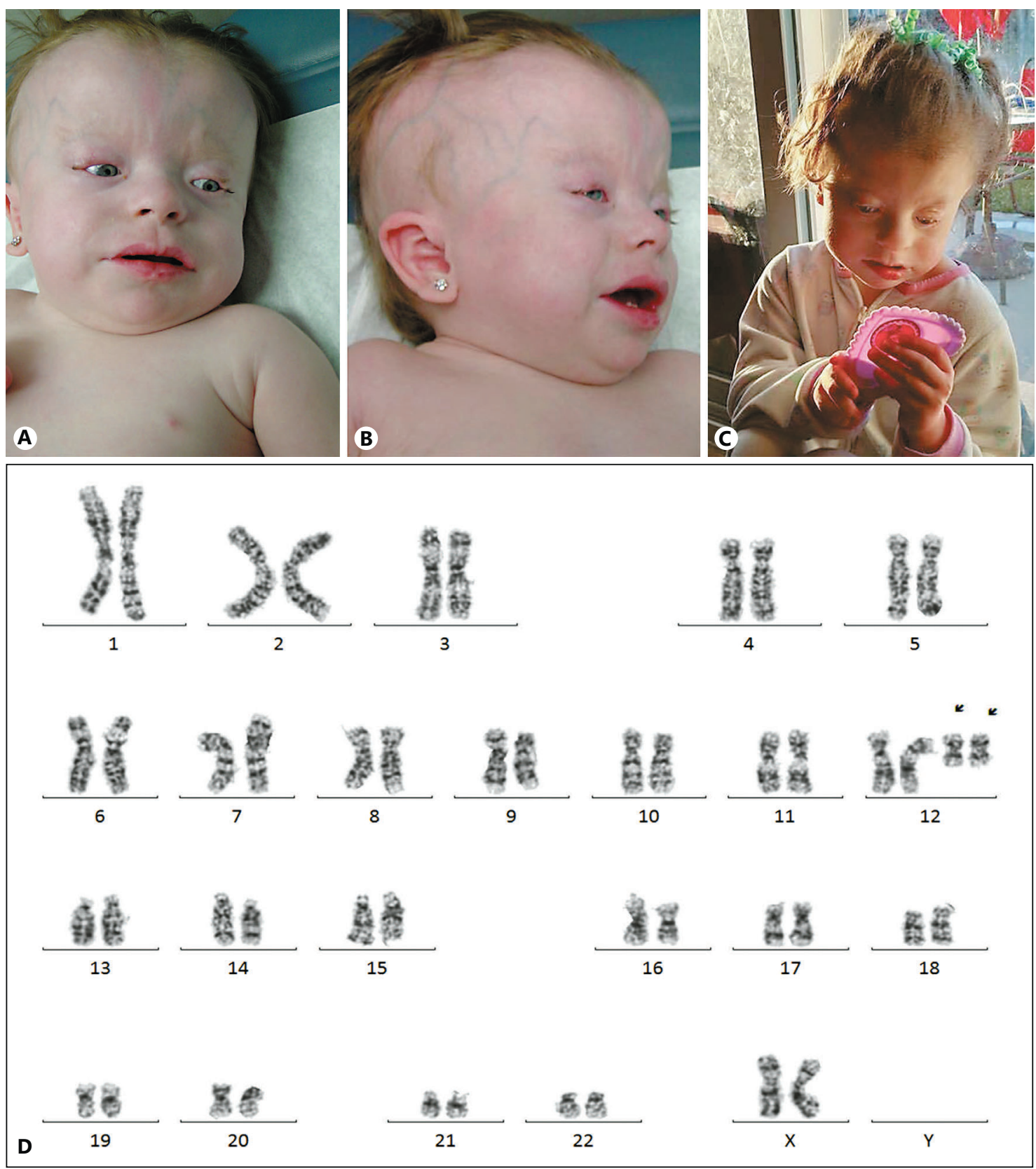

Fig. 1. A-C The craniofacial characteristics as noted in text: prominent forehead, prominent scalp veins, sparse hair over the temples, upslanting palpebral fissures, absence of medial eyelashes, sparse eyebrows, malar flattening, micrognathia, overfolded helices, wide and short nasal bridge, anteverted nares, broad mouth, and long philtrum continuing into the vermillion border of the upper lip. Photographs were taken when the patient was 21 months old (A, B) and 9 years old (C). The karyotype from the skin biopsy showing a female chromosome complement with 2 copies of isochromosome 12p resulting in hexasomy (D).

2 isochromosomes causing hexasomy of $12 \mathrm{p}$ has been reported only 4 times [Van den Veyver et al., 1993; Choo et al., 2002; Vogel et al., 2009; Aliu et al., 2013]. Here, we present a 10 -year-old girl with PKS who showed hexasomy $12 p$ in her skin fibroblasts. In addition to the characteristic dysmorphic features, our patient also had seizures and developed cardiomyopathy, a rare finding in PKS. 
Table 1. Clinical features of patients with hexasomy $12 \mathrm{p}$

\begin{tabular}{|c|c|c|c|c|c|}
\hline & $\begin{array}{l}\text { Current } \\
\text { patient }\end{array}$ & $\begin{array}{l}\text { Van den Veyver } \\
\text { et al. [1993] }\end{array}$ & $\begin{array}{l}\text { Choo et al. } \\
{[2002]}\end{array}$ & $\begin{array}{l}\text { Vogel et al. } \\
\text { [2009] }\end{array}$ & $\begin{array}{l}\text { Aliu et al } \\
\text { [2013] }\end{array}$ \\
\hline Developmental delay & + & NA & + & mild & mild \\
\hline Growth delay & + & + & + & + & NA \\
\hline Areas of hypo/hyperpigmentation & + & NA & + & + & + \\
\hline Facial dysmorphism & + & + & + & + & + \\
\hline Cleft lip/palate & + & - & + & - & NA \\
\hline Micrognathia & + & + & - & + & NA \\
\hline Hypertelorism & + & + & - & + & + \\
\hline Broad nasal root & + & + & + & + & + \\
\hline Prominent forehead & + & + & + & + & + \\
\hline Sparse hair bilaterally & + & + & + & + & + \\
\hline Seizures & + & NA & - & - & NA \\
\hline Cardiomyopathy & + & NA & - & + & NA \\
\hline Hearing loss & - & NA & + & + & NA \\
\hline \multicolumn{6}{|l|}{ Percentage of hexasomy $12 p$} \\
\hline Blood (age tested) & $0(\mathrm{NB})$ & $0(\mathrm{NB})$ & $0(\mathrm{NB})$ & $0(\mathrm{NB})$ & 0 (4 ys) \\
\hline Skin (age tested) & 70 (2 ys) & $95(\mathrm{NB})$ & $14.5(3 \mathrm{mo})$ & 20 (3 ys) & 46 (4 ys) \\
\hline
\end{tabular}

NA, not available/applicable; NB, newborn; mo, months; ys, years. Patients are included in this table if clinical features were described in detail. Another patient with hexasomy $12 \mathrm{p}$ was mentioned in the cohort reported by Conlin et al. [2012], however no clinical information was provided.

\section{Clinical Report and Results}

The proposita was born at $28^{1 / 2}$ weeks due to placental abruption. Her birth weight was $>90$ th centile, and she spent 69 days in the NICU for predictable preterm delivery complications, including premature lung disease, feeding problems requiring a nasogastric tube, sepsis, anemia, and jaundice.

Because of anal stenosis, she underwent serial anal dilations. Her dysphagia did not improve with the G-tube, and she had Nissen fundoplication surgery at 18 months of age. She was also identified as having a cleft palate and underwent surgical repair of the cleft palate during infancy.

As a 21-month-old female, she was referred for reevaluation by medical genetics because she was suspected of having an underlying syndrome. She was $<5$ th centile for height and weight, but had a normal OFC between the 25-50th centiles. Her craniofacial features included upslanting palpebral fissures, no medial eyelashes, sparse eyebrows, and lacrimal duct stenosis (Fig. 1A, B). She had a prominent forehead, prominent scalp veins and an unusual hair pattern (sparse over the temples), malar flattening, and micrognathia. Her helices were slightly overfolded, with very small external auditory canals. Her nose was distinctive with a wide, short nasal bridge, and anteverted nares. Her mouth was broad, and the philtrum was long, continuing into the vermillion border of the upper lip (sometimes referred as Pallister lip). She had delayed tooth eruption with only 2 central incisors of the mandible and wide alveolar ridges, which gave the palate a narrow appearance. The length of her arms was asymmetric, with the right arm being shorter than the left. Her right hand demonstrated a scar from resection of a preaxial digit. On the right hand, she had a single transverse crease and hypoplasia of the distal phalanges. She had patchy spots of rough skin on the right leg, stomach, arms, and face, but no clear areas of hyperpigmentation. Her neurological exam was notable for generalized hypotonia and irritability, and she was significantly delayed in all areas of pediatric development.

Genomic microarray analysis performed on a peripheral blood specimen was reported as normal.

The constellation of features: large birth weight, typical craniofacial features (especially Pallister lip), neurobehavioral profile, suggestions of mosaicism (asymmetry, patchy skin changes, retinal mottling), and normal cytogenetic studies from peripheral blood was highly suspicious for PKS.

A skin biopsy was obtained to perform cytogenetic studies from fibroblasts. The biopsy underwent standard tissue culture procedure, after which the cells were harvested and stained with Giemsa. The karyotype was reported as $48, \mathrm{XX},+\mathrm{i}(12)$ $(\mathrm{p} 10) \times 2[14] / 46, \mathrm{XX}[6]$ at the 400-band level (Fig. 1D).

Around the age of 2 years, an attempt was made to advance facial bones for the purpose of facial restoration and specifically to address her proptosis. Between the ages of 3 and 4 years, she had skin grafts to her eyelids to protect her cornea.

When she was about 3 years old, she developed epilepsy. The seizures were not well controlled with levetiracetam or lorazepam, but responded very well to zonisamide and nasal midazolam.

Chronic, recurrent upper respiratory infections necessitated placement of pressure equalization tubes, tonsillectomy, and mastoidectomy.

As of 10 years, her growth remains consistent and proportionate, but is below the 5 th centile for both height and weight (Fig. 1C). She sleeps well, but is unable to close her eyes when she is asleep. Unfortunately, this has continued to contribute to corneal erosions and scar tissue. She has developed a cardiomyopathy. She has 
had episodes of aspiration pneumonia. She continues to have gastroesophageal reflux as well as constipation. Her deciduous teeth have been affected by acid reflux. Her unilateral renal dysgenesis resulted in the loss of function of her right kidney, but there are no clinical features of any form of renal dysfunction.

While she has no classic pubertal changes, she has small patches of fine pubic hair but is otherwise Tanner stage 1 for sexual development. She is able to vocalize, but has minimal expressive language abilities. She can become easily overstimulated, which can result in seizure activity. In general, she is thought of as a happy child. She enjoys watching TV, physical contact with her family, and playing with toys.

\section{Discussion}

The clinical features seen in patients with PKS show a wide spectrum [Kostanecka et al., 2012; Blyth et al., 2015; Costa et al., 2015]. The most common findings include dysmorphic facial features, pigmentary irregularities of the skin, and hypotonia. Developmental delays and intellectual disabilities are also common. Some patients may have seizures. Structural cardiac defects have been reported; however, cardiomyopathy is rarely part of the clinical presentation [Tilton et al., 2014].

The $\mathrm{i}(12)(\mathrm{p} 10)$ is the diagnostic finding of PKS. Hexasomy $12 p$ due to 2 isochromosomes have been reported only 4 times [Van den Veyver et al., 1993; Choo et al., 2002; Vogel et al., 2009; Aliu et al., 2013]. The previously reported patients ranged in severity from a stillbirth with a severe phenotype to a young girl with a mild phenotype (Table 1). The current data are insufficient to conclude whether hexasomy $12 p$ carries a more severe phenotype than tetrasomy $12 p$ in a mosaic pattern.

It is difficult to establish a genotype-phenotype correlation in disorders with mosaic abnormalities. In most cases, gene content or gene dosage is not sufficient to predict outcome. In other words, the severity of symptoms cannot be predicted by either gene-dosage or the percentage of mosaic cells in the tissue examined [Hunter et al., 1985; Choo et al., 2002; Conlin et al., 2012; Spinner et al., 2014]. The tissue distribution of the mosaicism may represent a more critical factor, which is also difficult to assess [Hunter et al., 1985; Choo et al., 2002].

The molecular mechanism of $\mathrm{i}(12 \mathrm{p})$ formation in PKS is not completely understood; however it has been suggested that the primary error is prezygotic and most likely of maternal origin [Wenger et al., 1988; Struthers et al., 1999; Conlin et al., 2012].

Regardless of the mechanism of PKS expression and severity, it is important to diagnose PKS early, in order to optimize the management of the patients and to provide genetic counseling. Although characteristic clinical features of PKS exist, clinical diagnosis of this condition is often challenging [Aliu et al., 2013]. A complicating factor in the diagnosis process is the need of accurate cytogenetic analysis and the choice of specimen for analysis. Indeed, it is common to find a normal karyotype from blood chromosomes and mosaicism for the isochromosome in fibroblasts in patients with PKS [Van den Veyver et al., 1993; Choo et al., 2002; Vogel et al., 2009; Conlin et al., 2012].

The age of patients is a critical parameter in assessing the level of mosaicism. The younger patients tend to show a higher fraction of mosaic $\mathrm{i}(12 \mathrm{p})$ in peripheral blood, and the percentage of mosaicism decreases as the patient ages [Hunter et al., 1985; Choo et al., 2002; Conlin et al., 2012 ]. In addition, the fibroblasts retain the $i(12 p)$ for longer than hematopoietic cells, likely because of their slower turnover rate. In fact, in a study of 15 previously diagnosed PKS patients, Conlin et al. [2012] determined that the $\mathrm{i}(12 \mathrm{p})$ was detectable by microarray in $100 \%$ of fibroblast samples, but only $46 \%$ of blood samples.

The diagnosis of PKS can also be challenging due to technical limitations in the detection of $i(12 p)$. The use of genome-wide technologies such as SNP or CGH arrays has improved the diagnosis of PKS as indicated by several reported cases [Kostanecka et al., 2012; Blyth et al., 2015]. The clinical sensitivity of these technologies are significantly higher than the standard G-banding karyotyping in detection of mosaic abnormalities, such as i(12p). Unlike karyotype, microarray technologies do not require cell cultures. It has been proposed that wild type cells have a growth advantage in in vitro cultures over i $(12 p)$ cells. Since a few weeks of fibroblast cell culture are necessary to perform standard karyotyping banding, it is possible that the cell culture impairs the detection of the mosaicism, reducing the fraction of aneuploid cells.

\section{Conclusion}

The severity of PKS does not directly correlate with gene dosage or level of mosaicism. Furthermore, the choice of the tissue to be tested and the diagnostic technique complicates the detection of mosaicism. Therefore, it is critical for geneticists to be aware of such limitations in choosing the appropriate specimen type and genetic technology for diagnostic testing in patients presenting with symptoms indicative of PKS. 


\section{Acknowledgment}

We are indebted to the family for their participation.

\section{Statement of Ethics}

Informed consent and the permission for the publication of the accompanying images were obtained from the parents. The study was conducted according to the Declaration of Helsinki.

\section{Disclosure Statement}

The authors have no conflicts of interest to declare.

\section{Author Contributions}

R.M.T., S.T.S., and A.F.R. contributed to conception and design of the report. A.F.R. did the clinical evaluation. R.M.T., M.C.L., and S.T.S. performed and interpreted genetic analyses. R.M.T. and E.P. drafted, all authors reviewed, R.M.T. and A.F.R. edited the manuscript.

\section{References}

Aliu N, Rieubland C, Courage C, Mamavar M, Gallati S, Lemke J: Pallister-Killian syndrome due to mosaicism of two supernumerary isochromosomes (hexasomy 12p) in a girl with relatively mild phenotype (Poster). European Society of Human Genetics Congress, June 8-11, Paris, 2013.

Blyth M, Maloney V, Beal S, Collinson M, Huang $S$, et al: Pallister-Killian syndrome: a study of 22 British patients. J Med Genet 52:454-464 (2015).

Choo S, Teo SH, Tan M, Yong MH, Ho LY: Tissue-limited mosaicism in Pallister-Killian syndrome - a case in point. J Perinatol 22: 420-423 (2002).

-Conlin LK, Kaur M, Izumi K, Campbell L, Wilkens A, et al: Utility of SNP arrays in detecting, quantifying, and determining meiotic origin of tetrasomy 12p in blood from individuals with Pallister-Killian Syndrome. Am J Med Genet A 158A:3046-3053 (2012).
Costa LS, Zandona-Teixeira AC, Montenegro MM, Dias AT, Dutra RL, et al: Cytogenomic delineation and clinical follow-up of $10 \mathrm{Bra}-$ zilian patients with Pallister-Killian syndrome. Mol Cytogenet 8:43 (2015).

Hunter AG, Clifford B, Cox DM: The characteristic physiognomy and tissue specific karyotype distribution in the Pallister-Killian Syndrome. Clin Genet 28:47-53 (1985).

Kostanecka A, Close LB, Izumi K, Krantz ID, Pipan M: Developmental and behavioral characteristics of individuals with Pallister-Killian syndrome. Am J Med Genet A 158A:30183025 (2012).

-Schinzel A: Tetrasomy 12p (Pallister-Killian syndrome). J Med Genet 28:122-125 (1991).

-Spinner NB, Conlin LK: Mosaicism and clinical genetics. Am J Med Genet C Semin Med Genet 166C:397-405 (2014).

Struthers JL, Cuthbert CD, Khalifa MM: Parental origin of the isochromosome 12p in PallisterKillian syndrome: molecular analysis of one patient and review of the reported cases. Am J Med Genet 84:111-115 (1999).
Tilton RK, Wilkens A, Krantz ID, Izumi K: Cardiac manifestations of Pallister-Killian syndrome. Am J Med Genet A 164A:1130-1135 (2014).

-Van den Veyver IB, Macha ME, McCaskill C, Carpenter RJ, Shaffer LG: Prenatal diagnosis and clinical findings in a case of hexasomy 12p. Am J Med Genet 47:1171-1174 (1993).

Vogel I, Lyngbye T, Nielsen A, Pedersen S, Hertz JM: Pallister-Killian syndrome in a girl with mild developmental delay and mosaicism for hexasomy 12p. Am J Med Genet A 149A:510514 (2009).

-Wenger SL, Steele MW, Yu WD: Risk effect of maternal age in Pallister $\mathrm{i}(12 \mathrm{p})$ syndrome. Clin Genet 34:181-184 (1988).

Wilkens A, Liu H, Park K, Campbell LB, Jackson $\mathrm{M}$, et al: Novel clinical manifestations in Pallister-Killian syndrome: comprehensive evaluation of 59 affected individuals and review of previously reported cases. Am J Med Genet A 158A:3002-3017 (2012). 